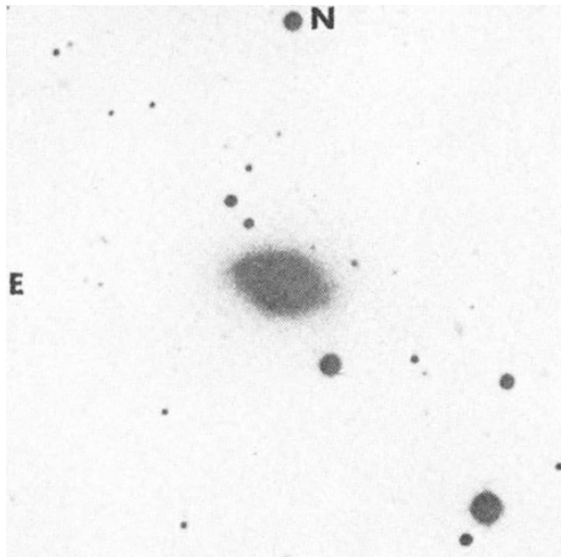

Galaxy NGC 7413. The QSO identified with the radio object $3 \mathrm{C} 455$ is the nearest starlike image to the north-east of the galaxy, separated from it by roughly 23" (from Astrophys. J. Lett., 171, plate L3; 1972).

be taken as indicating an association between the two objects, and a few years ago the great difference between their respective redshifts would have been accepted by most astronomers as conclusively against such an association. But there is now considerable evidence that quasars and galaxies are associated, and that the redshiftdistance relation is not universally applicable (see, for example, Jaakkola, Nature, 234, 534; 1971).

It is particularly significant in the present context that four $3 \mathrm{C}$ QSOs are already known to lie within a few arc seconds of bright galaxies (Burbidge et al., Astrophys. J., 170, 233; 1971); even this small number of associations is unlikely to arise from a chance superposition on the sky of unrelated objects, and the discovery of another such system greatly strengthens the position of those cosmologists who argue for a real physical link between QSOs and galaxies.

\section{PEST CONTROL}

\section{Doing without Chemicals}

from a Correspondent

From January 4 to 7 , members of the British Ecological Society, the Association of Applied Biologists and of the Pesticide Group of the Society of Chemical Industry met in Oxford for a symposium entitled "Increasing the Biological Contribution to the Control of Pests and Diseases". Although ecologists and even applied biologists might be expected to hold the point of view implicit in this title-that increasing the biological control of pests is desirable--it is encouraging to find industrial chemists, who have a vested interest in chemical control, and who could therefore look on the replacement of chemical pesticides by other means as a threat to their livelihood, also participating in this symposium. It was soon clear that the term "biological control" meant different things to different speakers. Some spoke of using parasites and predators to coritiol insect pests, some discussed methods of husbandry which reduced infestation. Sterilization and genetic engineering were also advocated. The only thing in common was the negative implication that biological control meant not relying solely on chemical pesticides, whether insecticides, fungicides, nematicides or herbicides, to control crop damage, or to prevent the spread of insect-borne diseases. It was also apparent that few of the speakers foresaw any likelihood of the total abandonment of chemical controls, at least in the near future, though many expressed the hope that safer chemicals would be used more scientifically.

On the first day a series of contributions attempted to provide the ecological background to the symposium. Thus, Dr J. P. Dempster (Monks Wood Experimental Station) and $\mathrm{Mr} \mathrm{T}$. H. Coaker (University of Cambridge) described field experiments with different spacing of brassica crops, and using various types of undersowing, on the levels of pest insects and their enemies. Professor G. C. Varley (University of Oxford) discussed his classical observations to determine the key factors controlling insect numbers in an oak wood. Though this type of work already illuminates the process of pest control, it has at present little application to agricultural problems.

The keynote address was then given by $\mathrm{Dr} \mathrm{F}$. Wilson (CSIRO, Australia) who drew on his unrivalled personal experience of the use of parasitic and predatory insects to control pest species. It was revealing, however, that he still found it useful to quote such long. established successes of biological control as that of the cottony-cushion scale in California by the vedalia beetle (1888), of the prickly pear by the caterpillar of Cactoblastis in Australia (1925) and of the coconut moth in Fiji by the tachinid parasite (1925). He made it clear, however, that even if these successes had seldom been equalled by later workers, real progress continued in many areas, continental as well as islands, in spite of the tiny financial investment in this type of work.

"Classical" biological control, that is the use of parasites and predators, has been useful in agriculture, but has never been effective against the vector of a human disease. Other non-chemical techniques, however, are showing promise. Dr G. Davidson (London School of Hygiene and Tropical Medicine) discussed such topics as genetical control, and the introduction of individuals with cytoplasmic incompatibilities which may give rise to widespread sterility in mosquitoes. It is hoped that such techniques will be useful in areas where disease vectors have become resistant to insecticides.

\title{
New Ways with Gel Chromatography
}

THE theory and practice of transport processes, particularly ultracentrifugation and gel column chromatography, applied to the analysis of dissociating macromolecular systems, have been worked out largely by Gilbert and his associates. This approach is proving of increasing value for the measurement of subunit equilibria in proteins, these being of direct relevance to cooperative interactions.

In next Wednesday's Nature New Biology (January 26) Gilbert et al. describe an interesting modification of the method, to allow a direct quantitative comparison between the equilibrium constants for two systems in dissociation equilibrium. The analysis has been applied to met- and oxyhaemoglobin. The procedure consists in equilibrating a gel column with one of the components, such that the concentration in the effluent is constant, and then switching to the other component at the identical concentration. By monitoring the effluent at an isosbestic point for the two derivatives, the elution profile can be determined. If now the position of the equilibrium (dimer-tetramer in the case of haemoglobin) is the same in both species, the concentration plateau will remain. If it is not, there will be a perturbation, the shape of which is determined by the dimer and tetramer elution volumes and the ratio of the dissociation constants. For the system in question this ratio is not in fact unity, and by a least-squares fit to the observed profile it can be determined with excellent precision. The corresponding free energies of dissociation for oxy- and methaemoglobin differ by about $1.1 \mathrm{kcal} \mathrm{mole}^{-1}$.

An extension of the same approach can be used to determine the relative equilibrium constants for the formation of hybrids, that is to say tetramers containing two oxyhaemoglobin and two methaemoglobin subunits. This equilibrium can be observed by first equilibrating the column with oxyhaemoglobin. and following this with a mixture of both derivatives at the same total concentration. The equilibrium constant for heterotetramer formation turns out to be almost exactly the geometric mean of those for the dissociation of oxyhaemoglobin and of methaemoglobin, from which it follows that essentially complete hybridization is to be expected. This is in accord with early observations on the species present after partial oxidation of oxyhaemoglobin. 